\title{
Subjective vs. documented reality: A case study of long-term real-life autobiographical memory
}

\author{
Avi Mendelsohn, Orit Furman, Inbal Navon, and Yadin Dudai ${ }^{1}$ \\ Department of Neurobiology, The Weizmann Institute of Science, Rehovot 76100, Israel
}

\begin{abstract}
A young woman was filmed during $2 \mathrm{~d}$ of her ordinary life. A few months and then again a few years later she was tested for the memory of her experiences in those days while undergoing fMRI scanning. As time passed, she came to accept more false details as true. After months, activity of a network considered to subserve autobiographical memory was correlated with memory confidence rather than with accuracy. After years, mainly regions of the temporal pole displayed this pattern. These results might reflect a slow process of increased reliance on schemata at the expense of accuracy.
\end{abstract}

Autobiographical memory (AM) is the recollection of self-relevant information about personal life history (Conway and Holmes 2004). AM research is commonly hindered by lack of reliable documentation of the targeted events (Cabeza and Jacques 2007). Here we present a case study of E.S., a healthy 29 yr-old woman, who was filmed during ordinary life activities and tested months and years later while in the MRI scanner for her recollection of the documented experiences.

E.S. agreed to serve as the protagonist in a documentary film produced for use in behavioral and neuroimaging studies in our lab. She was filmed during two regular working days, from which $10 \mathrm{~h}$ of footage were collected. E.S. was filmed while carrying out her daily activities at home and at work, such as talking on the phone, preparing lunch, teaching drama classes at high school, watching TV, and rollerblading with friends. None of the events was directed, scripted, or preplanned by the filming crew members (minor technical requests notwithstanding). Furthermore, E.S. was unaware of the possibility that her own memory would be tested. The footage was edited into a movie that was used in memory experiments (Mendelsohn et al. 2008). Only at a later stage did we decide to test E.S. on her own memory of the documented events.

E.S. answered computerized memory questionnaires that targeted details from the filmed events while undergoing fMRI scanning on two time points, one after 4 mo (Recent), the other after $2 \mathrm{yr}$ and 5 mo (Remote). A Verbal Questionnaire (VQ) was administered in both the Recent and Remote scans, and a Pictorial Questionnaire (PQ) was administered only in the Remote scan. Both questionnaires were administered using Presentation software (Neurobehavioral Systems). VQ consisted of 51 yes/no questions, probing memory for documented events (e.g., During recess, did one of your students complain about an audition? Did your neighbor open the door without his shirt on?). Some questions contained accurate details, while others contained altered details. In addition, E.S. was required to rate her confidence on each answer as either "high," "medium," or "low." A fixation point on a blank screen was presented for 6-8 sec between questions. Memory performance and confidence rating were recorded for each question. PQ consisted of 80 test items, each comprising a cued-recall phase followed by a recognition phase. In the cued-recall phase, a still image from the movie appeared on

\footnotetext{
'Corresponding author.
}

E-mail Yadin.dudai@weizmann.ac.il; fax 972-8-946-9244.

Article is online at http://www.learnmem.org/cgi/doi/10.1101//m.1157709. screen for $4 \mathrm{sec}$, accompanied by a question targeting a particular content detail. E.S.'s task was to use the visual cue in order to recall the scene and generate the answer to the question. In the recognition phase, the picture and question were presented again, this time with two possible answers. E.S. was asked to choose one answer and rate her confidence in that choice. Both image and text remained on screen until a response was recorded. An additional perceptual control task was included, in which a scrambled movie image was presented for $4 \mathrm{sec}$, after which the subject was to indicate which part of the image (left or right) was brighter.

The Recent scan was performed on a 3T GE Signa Horizon echo speed scanner at Sourasky Medical Center, Tel Aviv, Israel. The Remote scan was performed on a 3T Trio Magnetom Siemens scanner, at the Weizmann Institute of Science, Rehovot, Israel. The protocols were approved by the Institutional Review Board of the Sourasky Medical Center. Three-dimensional T1-weighted anatomical images were acquired with high-resolution 1-mm slice thickness (Recent scan: SPGR echo sequence, TR $40 \mathrm{~ms}$, TE $9 \mathrm{~ms}$, $0.93 \times 0.93 \times 1$-mm voxels; Remote scan: $3 \mathrm{D}$ MP-RAGE sequence, TR $2300 \mathrm{~ms}$, TE $2.98 \mathrm{~ms}, 1 \times 1 \times 1$-mm voxels). In the Recent scan, T2*-weighted echo planar imaging (EPI) images were acquired (TR $2000 \mathrm{~ms}$, TE $40 \mathrm{~ms}$, flip angle $80^{\circ}, 32$ oblique slices without gap, $15^{\circ}$ from ACPC, $3.125 \times 3.125 \times 4-\mathrm{mm}$ voxel). The parameters of the Remote scan were as follows: TR $2000 \mathrm{~ms}$, TE $30 \mathrm{~ms}$, flip angle $80^{\circ}, 35$ oblique slices without gap, $30^{\circ}$ from ACPC, $3 \times 3 \times 4-\mathrm{mm}$ voxel. The functional MRI data from both VQ scans (Recent and Remote), as assessed by the "general" model (see below), unveiled extensive overlap of brain activity (data not shown).

Data from both scans were preprocessed, spatially normalized, and superimposed onto 3D anatomical volume sets using BrainVoyager QX 1.8.6 (Brain Innovation) as described in Mendelsohn et al. (2008). Analysis of the PQ functional data set (obtained at Remote) was performed by constructing a General Linear Model (GLM). Because AM recollection and resting states often engage the same core brain networks (Spreng et al. 2008), the perceptual condition, rather than fixation, was subtracted from the recognition phase of the PQ data $(P<0.05$, corrected for multiple comparisons, cluster size $>150 \mathrm{~mm}^{3}$ ). Regions of interest (ROIs) defined on the resulting statistical parametric map were then used to explore brain activity during the VQ test in both Recent and Remote intervals.

Four GLMs were constructed to analyze the VQ test data separately for each of the two scans (Recent and Remote), and were used for explorative whole-brain comparisons in addition to 
functional- and anatomical-guided ROI analysis (see below). In GLM 1 ("general" model), all the questions in the test were treated as one condition. In GLM 2 ("veracity" model), the same questions were divided into "correct" and "incorrect" conditions based on E.S.'s answers. In GLM 3 ("confidence" model), the same questions were categorized by confidence ratings given by E.S. (high, medium, or low), regardless of answer accuracy. GLM 4 ("parametric" model) was used to assess voxel-by-voxel parametric changes in brain activity during the VQ test, which corresponded to parametric ratings of confidence. The model consisted of the same conditions as defined in the "general" model, along with a parametric regressor, for which a positive weight was assigned to questions with high confidence ratings, a zero weight to questions with medium confidence, and a negative weight to questions with low confidence ratings. To test for brain regions that were activated according to this subjective-memory strength pattern, the parametric condition was tested against baseline $(P<0.0001$, not corrected, $t>3.8$, cluster size $>150 \mathrm{~mm}^{3}$ ). In all GLMs the condition time vectors were convolved with a canonical hemodynamic response function (HRF), and six motion-correction parameters were added as covariate regressors.

Finally, an anatomy-guided ROI analysis was performed on bilateral hippocampi and amygdalae. These regions were selected based on their reported involvement in consolidation and retrieval of episodic memories (Gilboa et al. 2004; Cabeza and Jacques 2007; Viard et al. 2007). Data from the VQ test at both Recent and Remote interval scans were extracted for the above ROIs, using GLM 1 ("general" model) and GLM 3 ("confidence" model).

\section{The passage of time increases acceptance of wrong details}

On the Recent VQ test, E.S. answered $68.6 \%$ of the questions correctly, compared with $60.8 \%$ at the Remote interval. The reduction in memory accuracy between the two tests was attributed to an increase of $\sim 12 \%$ in "False alarm" (FA) errors in Remote compared with Recent interval (Recent 17.7\%, Remote 29.4\%; Table 1A). Conversely, "Miss" errors (judging true statements as false) showed the opposite trend (Recent 13.7\%, Remote 9.8\%). Taken together, E.S.'s response criterion $\left(-0.5^{*}[\mathrm{FA}\right.$ rate + Hit rate]) shifted at the Remote interval so as to generate more "yes" answers (response criterion: Recent 0.01 , Remote -0.4 ), resulting in decreased discriminability between old and new statements ( $d$ ' scores: Recent 0.97, Remote 0.7).

Concurrently, with an increasing proportion of "yes" answers, the proportion of high confidence ratings (regardless of accuracy) increased as well during Remote testing (Recent $41 \%$, Remote 55\%). The proportion of low confidence ratings decreased (Recent 35\%, Remote 21\%), whereas the proportion of medium confidence ratings remained stable across time (24\%). Table $1 \mathrm{~B}$ presents the percentage of correct and incorrect responses that were given high, medium, or low confidence ratings in each testing interval. A modest relationship is apparent between accuracy and confidence ratings, indicated by a large proportion of high confidence ratings for correct answers and a low proportion of such ratings for incorrect responses at both test intervals.

\section{Activity in the AM network is correlated with subjective sense of memory strength rather than with accuracy}

In analysis of the PQ test scan, subtracting activity during the perceptual task from activity during the recognition task unveiled a network of brain regions similar to that reported in previous AM studies (Gardini et al. 2006; Svoboda et al. 2006; Cabeza and Jacques 2007). This network included the precuneus, retrosplenial areas, posterior cingulate gyri, medial prefrontal cortex (mPFC), bilateral ventrolateral prefrontal cortex (VLPFC), bilateral dorsolateral cortex (DLPFC), bilateral temporo-parietal junction (TPJ), left parahippocampal gyrus (PHG), and temporal cortices. These regions then served as ROIs for extracting functional data from the VQ scans at both the Recent and Remote intervals (Fig. 1A).

For the above ROIs, $\beta$ values from the VQ data sets were extracted for correct and incorrect answers, using the "veracity" model. Activity was not markedly different between correct and incorrect events at both Recent and Remote time points (Fig. 1C). In addition, $\beta$ values were extracted from the same functional data of the aforementioned ROIs using the "confidence" model in order to explore the role of metamemory processes during retrieval. This analysis revealed a clear trend in practically all of the

Table 1. Memory performance and confidence ratings as a function of time

(A) Memory performance

\begin{tabular}{lcccccrr}
\hline & \multicolumn{3}{c}{ Correct answers } & & \multicolumn{2}{c}{ Incorrect answers } \\
\cline { 2 - 4 } & Hits & CR & Total & & Misses & FA & Total \\
\hline Recent & $29.4 \%$ & $39.2 \%$ & $68.6 \%$ & $13.7 \%$ & $17.7 \%$ & $31.4 \%$ \\
Remote & $33.3 \%$ & $27.5 \%$ & $60.8 \%$ & $9.8 \%$ & $39.2 \%$ \\
\hline
\end{tabular}

(B) Percentage of correct and incorrect responses for each confidence rating

\begin{tabular}{|c|c|c|c|c|c|c|}
\hline & \multicolumn{3}{|c|}{ Correct answers } & \multicolumn{3}{|c|}{ Incorrect answers } \\
\hline & Low & Medium & High & Low & Medium & High \\
\hline Recent & $25.7 \%$ & $22.9 \%$ & $51.4 \%$ & $56 \%$ & $25 \%$ & $19 \%$ \\
\hline
\end{tabular}

(A) Percentage of correct (left columns) and incorrect (right columns) answers in the Verbal Questionnaire (VQ) test at Recent and Remote intervals. Performance is divided into "Hit," "Correct rejection" (CR), "Miss," and "False alarm" (FA) responses. Total percentage of correct answers (sum of "Hit" and "CR") and incorrect answers (sum of "Miss" and "FA") are indicated for each test.

(B) Percentage of correct (left columns) and incorrect answers (right columns) that were given low, medium, or high confidence ratings in the VQ test at Recent and Remote intervals. Note that correct answers at both testing intervals were accompanied by more high confidence ratings compared with low and medium ones. In contrast, incorrect answers were associated with less high ratings than low or medium ones. 
A Regions of interest
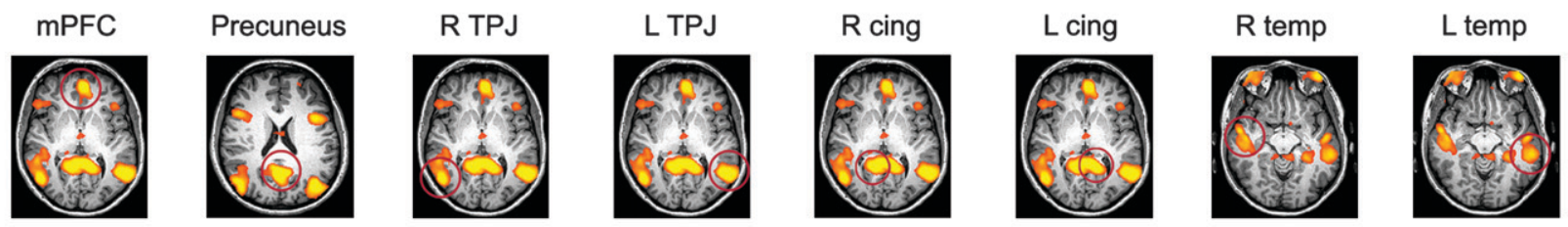

\section{B Confidence model}

Recent $\square$ Remote
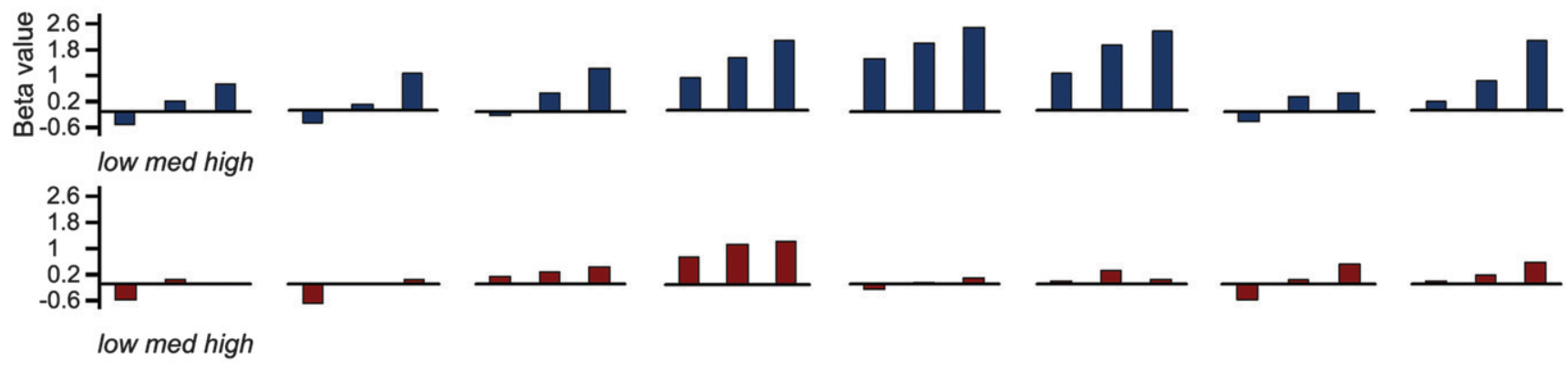

\section{Veracity model}
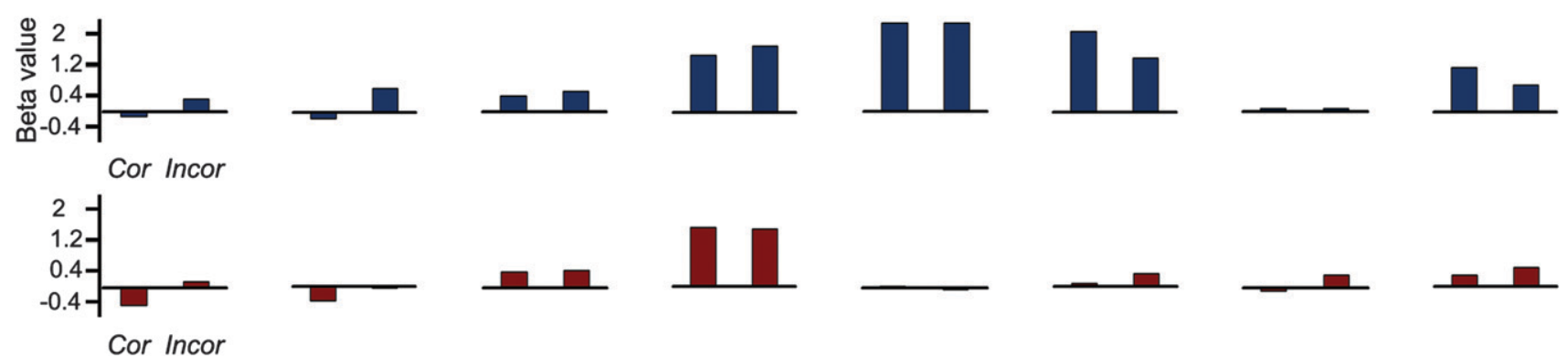

Figure 1. Correlation of accuracy and metamemory with activity in regions of interest. ( $A$ ) Regions of interest (ROIs) extracted from the Pictorial Questionnaire $(\mathrm{PQ})$ scan by contrasting recognition vs. perceptual task $\left(P<0.05\right.$, Bonferroni correction for multiple comparisons, cluster size $\left.>150 \mathrm{~mm}^{3}\right)$. Clusters of $64 \mathrm{~mm}^{3}$ were delineated around the peak activations of the circled ROI's and used for data analysis of the VQ scans. (R) Right; (L) left; (mPFC) medial prefrontal cortex; (cing) cingulate gyrus; (TPJ) temporo-parietal junction, (temp) temporal cortex. Brain images are shown in radiological orientation. (B) Functional data from the VQ scans of Recent (blue bars) and Remote (red bars) intervals, extracted from the ROIs delineated in $A$. $\beta$ values calculated from the "confidence" model are shown, reflecting activity for low, medium, and high confidence ratings. (C) $\beta$ values from the same ROls and scans as in $B$, calculated from the "veracity" model, divided into correct and incorrect responses.

aforementioned regions (Fig. 1B), in which increasing subjective memory strength (based on metamemory judgments) correlated with increased brain activity. Importantly, this trend was apparent mainly in the Recent period and much less so in the Remote scan. In summary, activity in the AM network was correlated with the subjective sense of memory strength rather than with memory accuracy, an effect that was primarily pronounced in the Recent interval.

\section{The passage of time restricts recollection-related activity in the AM network mainly to the temporal pole}

To complement the above metamemory finding, we used a voxelby-voxel parametric analysis on the VQ data using confidence ratings from each scanning interval. We found that in the Recent scan, the parametric analysis reinforced the outcome of the ROI analysis, yielding activity in several regions of the AM network. This network included the precuneus, MPFC, left VLPFC, bilateral TPJ, and bilateral DLPFC (Fig. 2, top). In the Remote scan, this analysis yielded activation primarily in bilateral temporal poles and left TPJ, with no activity found in other AM-network regions (Fig. 2, bottom). Importantly, in an explorative attempt to examine the comparison between correct and incorrect answers using the "veracity" model, differential activation in Recent was restricted to a small cluster in the left temporal lobe (centered in the white matter near BA 38), and none was detected in Remote.

In the anatomically guided ROI analysis of the hippocampi, activity was found to decrease in Remote compared with Recent scans (Fig. 3A). A trend for parametric activity related to subjective confidence rating (using the "confidence" model) was found only in the Recent scan (Fig. 3B). A similar effect was observed in bilateral amygdala as well (data not shown). 

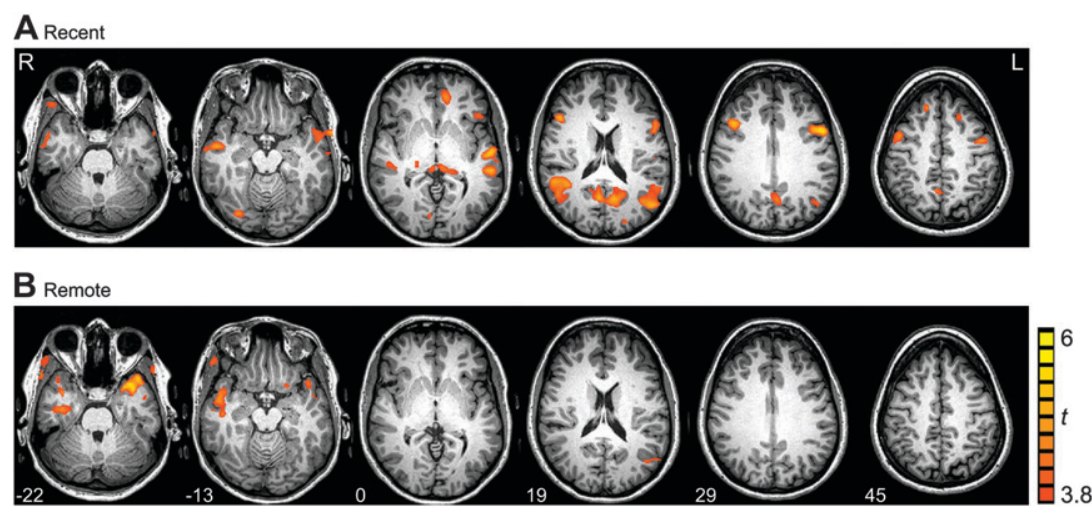

Figure 2. Parametric analysis of $\mathrm{VQ}$ data based on metamemory judgments. (A) Statistical maps here and in $B$ below show the outcome of a contrast between the parametric confidence regressor and fixation baseline, as defined in the "parametric" model $(P<0.0001$, uncorrected, cluster size $>150$ $\mathrm{mm}^{3}$ ). Parametric confidence effects for Recent are observed in left medial prefrontal cortex (BA10)/ anterior cingulate, bilateral precuneus (BA 31), bilateral temporo-parietal junctions (BA 39/19), left ventrolateral prefrontal cortex (BA 47), bilateral dorsolateral prefrontal cortex (BA 46/9), and bilateral middle temporal gyri (BA 21/22). (B) Parametric confidence effects for Remote interval are shown in bilateral temporal poles (BA 38), right middle temporal lobe (BA 21), and left TPJ (BA 39).
Case studies of cognitively intact individuals (often the investigator himself) were common in the early days of experimental psychology (Galton 1879; Ebbinghaus 1885), but later became the gold standard in the study of amnesic patients only (Scoville and Milner 1957; Stefanacci et al. 2000; Kritchevsky et al. 2004; Rosenbaum et al. 2005). However, the potential usefulness should not be neglected of case studies of real-life autobiographical memory of healthy individuals when the opportunity arises to combine extensive objective documentation of their unique experiences with memory tests and functional neuroimaging after long periods of time. E.S. provided us with just such an opportunity. When her life routines were filmed over $2 \mathrm{~d}$, E.S. did not even contemplate the possibility that her own memory would be challenged. And when tested after a few months, she thought that we would finally forget about her. We did not.

E.S.'s memory performance deteriorated somewhat between months and years. It is noteworthy that this deterioration is attributed here to commission errors, i.e., endorsing altered details from the events as true. This finding is in agreement with a previous diary study (Barclay and Wellman 1986), and may be construed as resulting from the mental ontogenesis of schemata that augment the tendency to accept false details at the expense of accuracy (Brewer 1996; Gilboa 2004). Although the possibility cannot be excluded that responses at Remote might have been influenced by the past encounter with the same questions, we find this unlikely given the long interval between the tests, the fact that both tests came as a surprise to E.S., and the observation that experience on the first test did not improve performance on the second.

Our data support earlier laboratorysetting findings of a neural network in which activity is correlated with recollection of AM memories, the "AM network" (Svoboda et al. 2006; Spreng et al. 2008). We find, however, that regions of this network are more sensitive to metamemory processes than to accuracy, i.e., sensitive to subjective confidence in retrieval success rather than to objective retrieval success. For generating metamemory judgments of AM, individuals tend to rely on perceptual vividness of the retrieved information (Talarico and Rubin 2003). Thus, the extent of vividness associated with recollection of AM might affect the sense of confidence regarding retrieval success. It was recently suggested that regions implicated in AM retrieval might have a broader role in cognition. Activity in core regions of this net-work are correlated with reconstruction of complex visual scenes (Hassabis and Maguire 2007) as well as with resting states commonly referred to as the "default mode" (Buckner et al. 2008; Spreng et al. 2008). Taken together, AM-network activity may reflect the extent to which one is able to mentally reconstruct detailed past events irrespective of accuracy. As years passed, confidence ratings were no longer reflected in the widespread AM-network activity, but rather in bilateral temporal poles and left TPJ. Corroborating the shift in response criterion, activity in temporal-pole regions could imply a qualitative change in recollection experience and accuracy judgment of AM. We suggest that with time, recollection of AM comes to rely to a higher extent on personal-semantic knowledge rather than on vivid recollection of the events. This notion is supported by previous research, implicating the temporal pole (Olson et al. 2007; Patterson et al. 2007), as well as the TPJ (Svoboda et al. 2006) in processing personal-semantic information.

A longstanding issue is the role of the hippocampus in memory consolidation and retrieval (Dudai 2004). Some authors consider the hippocampus essential for consolidation and for retrieval of recent but not remote declarative memories (Squire
A “General" model

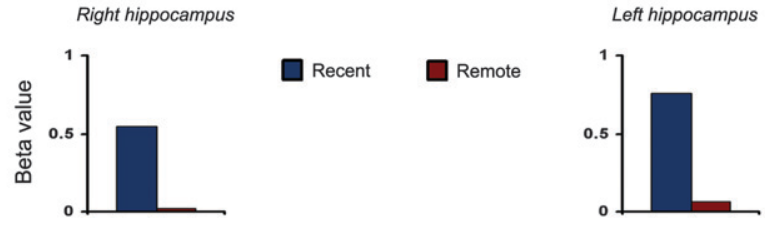

B "Confidence" model Right hippocampus
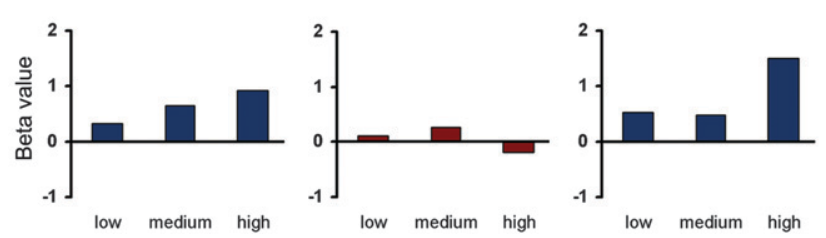

eft hippocampus

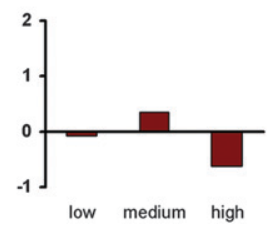

Figure 3. Hippocampal activity in Recent and Remote tests. Data are shown for right and left hippocampus (Talairach coordinate of ROI center: right hippocampus $-x=30, y=-22, z=-8$; left hippocampus $-x=-28, y=-22, z=-12$ ). (A) $\beta$ values calculated from BOLD data of right and left hippocampus, extracted using the "general" model in Recent (blue) and Remote (red) VQ scans. (B) $\beta$ values calculated from BOLD data of right and left hippocampus extracted using the "confidence" model in Recent (blue) and Remote (red) VQ scans, depicting activation during retrieval task for low, medium, and high confidence. 
et al. 2004; Kirwan et al. 2008). Others propose that the hippocampus remains vital in recollection (Nadel and Moscovitch 1997; Rosenbaum et al. 2008), provided the remote memories are reexperienced vividly (Gilboa et al. 2004; Viard et al. 2007; Piolino et al. 2008). Since AM recollection tends to lose its vividness over time (Gilboa et al. 2004; Piolino et al. 2008), we contend that the attenuation in hippocampal activity might be attributed to reduced vividness in recollection. This is in line with the aforementioned suggestion that remote memory comes to rely more extensively on personal semantics. Hence our results imply that high confidence of AM does not necessarily correlate with vividness; while the hippocampus seems to be needed for vivid AM recollection (Gilboa et al. 2004), it might not be needed for confidence judgments of AM recollection that lacks vividness.

In conclusion, E.S.'s memory performance unveiled a shift in response criterion, leading to endorsement of more false details as true as the years passed. Brain activity in her "AM network" did not discern accurate from inaccurate performance, but was sensitive to the confidence she had in her memory. After almost 2-1/2 $\mathrm{yr}$, this parametric activity was apparent primarily in her temporal poles, but not in typical AM network regions. All together, these findings may reflect the fact that over the years, E.S.'s mental autobiography acquired a more schematic flavor.

\section{Acknowledgment}

This research was supported by a grant from the Israeli Science Foundation, Jerusalem.

\section{References}

Barclay, C.R. and Wellman, H.M. 1986. Accuracies and inaccuracies in autobiographical memories. J. Mem. Lang. 25: 93-103.

Brewer, W.F. 1996. What is recollective memory? In Remembering our past—studies in autobiographical memory (ed. D.C. Rubin), pp. 19-66. Duke University, Hillsborough, NC.

Buckner, R.L., Andrews-Hanna, J.R., and Schacter, D. 2008. The brain's default network: Anatomy, function, and relevance to disease. Ann. N. Y. Acad. Sci. 1124: 1-38.

Cabeza, R. and Jacques, P.S. 2007. Functional neuroimaging of autobiographical memory. Trends Cogn. Sci. 11: 219-227.

Conway, M.A. and Holmes, A. 2004. Psychosocial stages and the accessibility of autobiographical memories across the life cycle. J. Pers. 72: 461-480.

Dudai, Y. 2004. The neurobiology of consolidations, or, how stable is the engram? Annu. Rev. Psychol. 55: 51-86.

Ebbinghaus, H. 1885. Ueber das Gedachtnis. Translated as Memory, a contribution to Experimental Psychology, 1913. Republished 1964, Dover, New York.

Galton, F. 1879. Psychometric experiments. Brain 2: 149-162.

Gardini, S., Cornoldi, C., De Beni, R., and Venneri, A. 2006. Left mediotemporal structures mediate the retrieval of episodic autobiographical mental images. Neuroimage 30: 645-655.

Gilboa, A. 2004. Autobiographical and episodic memory-one and the same? Evidence from prefrontal activation in neuroimaging studies. Neuropsychologia 42: 1336-1349.
Gilboa, A., Winocur, G., Grady, C.L., Hevenor, S.J., and Moscovitch, M. 2004. Remembering our past: Functional neuroanatomy of recollection of recent and very remote personal events. Cereb. Cortex 14: 12141225 .

Hassabis, D. and Maguire, E.A. 2007. Deconstructing episodic memory with construction. Trends Cogn. Sci. 11: 299-306.

Kirwan, C.B., Bayley, P.J., Galvan, V.V., and Squire, L.R. 2008. Detailed recollection of remote autobiographical memory after damage to the medial temporal lobe. Proc. Natl. Acad. Sci. 105: 2676-2680.

Kritchevsky, M., Chang, J., and Squire, L.R. 2004. Functional amnesia: Clinical description and neuropsychological profile of 10 cases. Learn. Mem. 11: 213-226.

Mendelsohn, A., Chalamish, Y., Solomonovitch, A., and Dudai, Y. 2008. Mesmerizing memories: Brain substrates of episodic memory suppression in posthypnotic amnesia. Neuron 57: 159-170.

Nadel, L. and Moscovitch, M. 1997. Memory consolidation, retrograde amnesia and the hippocampal complex. Curr. Opin. Neurobiol. 7: 217227.

Olson, I.R., Plotzker, A., and Ezzyat, Y. 2007. The enigmatic temporal pole: A review of findings on social and emotional processing. Brain 130: 17181731.

Patterson, K., Nestor, P.J., and Rogers, T.T. 2007. Where do you know what you know? The representation of semantic knowledge in the human brain. Nat. Rev. Neurosci. 8: 976-987.

Piolino, P., Desgranges, B., Hubert, V., Bernard, F.A., Matuszewski, V., Chételat, G., Baron, J.C., and Eustache, F. 2008. Reliving lifelong episodic autobiographical memories via the hippocampus: A correlative resting PET study in healthy middle-aged subjects. Hippocampus 18: $445-459$.

Rosenbaum, R.S., Köhler, S., Schacter, D.L., Moscovitch, M., Westmacott, R., Black, S.E., Gao, F., and Tulving, E. 2005. The case of K.C.: Contributions of a memory-impaired person to memory theory. Neuropsychologia 43: 989-1021.

Rosenbaum, R.S., Moscovitch, M., Foster, J.K., Schnyer, D.M., Gao, F., Kovacevic, N., Verfaellie, M., Black, S.E., and Levine, B. 2008. Patterns of autobiographical memory loss in medial-temporal lobe amnesic patients. J. Cogn. Neurosci. 20: 1490-1506.

Scoville, W.B. and Milner, B. 1957. Loss of recent memory after bilateral hippocampal lesions. J. Neurol. Neurosurg. Psychiatr. 20: 11-21.

Spreng, R.N., Mar, R.A., and Kim, A.S.N. 2008. The common neural basis of autobiographical memory, prospection, navigation, theory of mind and the default mode: A quantitative meta-analysis. J. Cogn. Neurosci. 1-20. doi:10.1162/jocn.2008.21029.

Squire, L.R., Stark, C.E.L., and Clark, R.E. 2004. The medial temporal lobe. Annu. Rev. Neurosci. 27: 279-306.

Stefanacci, L., Buffalo, E.A., Schmolock, H., and Squire, L.R. 2000. Profound amnesia after damage to the medial temporal lobe: A neuroanatomical and neuropsychological profile of patient E.P. J. Neurosci. 20: 70247035 .

Svoboda, E., McKinnon, M.C., and Levine, B. 2006. The functional neuroanatomy of autobiographical memory: A meta-analysis. Neuropsychologia 44: 2189-2208.

Talarico, J.M. and Rubin, D.C. 2003. Confidence, not consistency, characterizes flashbulb memories. Psychol. Sci. 14: 455-461.

Viard, A., Piolino, P., Desgranges, B., Chételat, G., Leberton, K., Landeau, B., Young, A., De La Sayette, V., and Eustache, F. 2007. Hippocampal activation for autobiographical memories over the entire lifetime in healthy aged subjects: An fMRI study. Cereb. Cortex 17: $2453-2467$.

Received July 12, 2008; accepted in revised form November 20, 2008. 


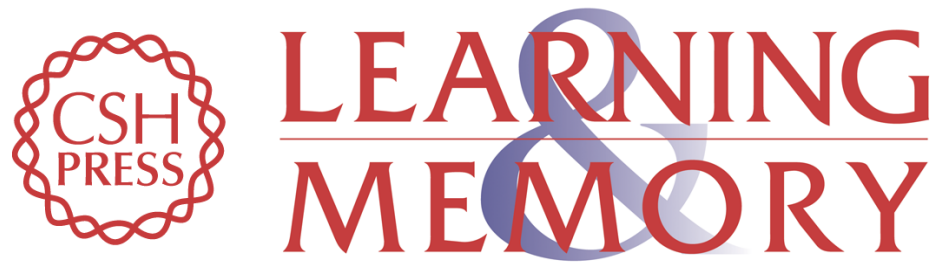

\section{Subjective vs. documented reality: A case study of long-term real-life autobiographical memory}

Avi Mendelsohn, Orit Furman, Inbal Navon, et al.

Learn. Mem. 2009, 16:

Access the most recent version at doi:10.1101//m.1157709

References This article cites 25 articles, 4 of which can be accessed free at:

http://learnmem.cshlp.org/content/16/2/142.full.html\#ref-list-1

License

Email Alerting Receive free email alerts when new articles cite this article - sign up in the box at the Service top right corner of the article or click here. 